\title{
USO DE HISTÓRIA DA MATEMÁTICA NO ENSINO MÉDIO: UMA ABORDAGEM INICIAL PARA O ENSINO DA TEORIA DOS GRAFOS
}

\author{
Lauro Chagas e Sá1, Sandra Aparecida Fraga da Silva²
}

\author{
Coordenadoria do Curso de Licenciatura em Matemática \\ Programa de Pós-graduação em Educação em Ciências e Matemática \\ Instituto Federal do Espírito Santo - Campus Vitória \\ Avenida Vitória, 1729 - Jucutuquara, Vitória, Espírito Santo. CEP 29040780.
}

\section{RESUMO}

Em 2009, o Currículo do Estado do Espírito Santo - Brasil foi alterado, incluindo tópicos da Teoria Grafos para o segundo e o terceiro anos do Ensino Médio. Face à inclusão curricular, emerge a necessidade de investigar atividades que possam discutir esse tema na Educação Básica. $\mathrm{O}$ objetivo deste trabalho foi analisar uma abordagem inicial da Teoria dos Grafos por meio do uso de um problema histórico real e propondo uma situação hipotética à luz da Teoria dos Grafos. 0 problema fictício foi elaborado para associar o Problema das Sete Pontes de Konisberg à realidade física do município, que é chamado de "Problema das Seis Pontes de Vitória", sendo aplicado aos alunos do segundo ano de ensino médio de uma escola pública do município de Vitória do Estado do Espírito Santo - Brasil, com resultados satisfatórios.

Palavras-chave: teoria dos grafos. história da matemática. Euler. ensino médio.

\section{ABSTRACT}

In 2009, the curriculum of the State of Espirito Santo - Brazil has changed, including topics of theory graphs for the second and third years of high school. Given the curricular inclusion, emerges the need to investigate activities that may discuss this topic in Basic Education. The objective of this study was to analyze an initial approach Graph Theory through the use of a real historical problem and proposing a hypothetical situation in light of Graph Theory. The fictitious problem was designed to associate the problem of the Seven Bridges Konisberg to physical reality of municipality, which is called "Issue Six Bridges of Vitória" being applied to students of the second year of high school at a public school in the municipality of Vitória in the State of Espirito Santo - Brazil, with satisfactory results.

Palavras-chave: graph theory, history of mathematics, Euler; high school.

\footnotetext{
1 Aluno do Curso de Licenciatura em Matemática Instituto Federal do Espírito Santo e bolsista de Iniciação Científica do Fundo de Apoio à Ciência e Tecnologia do Município de Vitória (FACITEC-Vitóra). E-mail: lauro_sa@live.com

2 Professora de Educação Matemática do Instituto Federal do Espírito Santo, D.Ed. - Docente do Curso de Licenciatura em Matemática e do Programa de Pós-graduação em Educação em Ciências e Matemática do Ifes. Email: sfraga@ifes.edu.br
} 


\section{INTRODUÇÃo}

O desenvolvimento de teorias matemáticas que relacionam elementos de conjuntos discretos é bastante recente se comparado à história da "matemática contínua". Exemplo disso é a Teoria dos Grafos, formulada já no século XVIII e que ainda assim foi "redescoberta muitas vezes" (HARARY, 1973, apud BOAVENURA NETTO, 2006, p. 2). Além disso, o desenvolvimento dessa teoria foi impulsionada somente no Século XX, pelos problemas de otimização no campo da pesquisa operacional, já que, até então, suas aplicações eram feitas em áreas disjuntas, como circuitos elétricos e química orgânica.

Nas últimas décadas, pesquisas da área de Educação e Educação Matemática (BRIA, 2001; MUNIZ JUNIOR, 2007; MALTA, 2008; DEGGERONI, 2010; GUALANDI, 2012) têm defendido a inserção da Teoria dos Grafos no Ensino Fundamental e Médio, como viés para discussão de problemas de matemática aplicada ao cotidiano escolar. Nesse cenário, Currículo Básico da Escola Estadual do Espírito Santo (ESPÍRITO SANTO, 2009), passou a prever o ensino da Teoria dos Grafos nos dois últimos anos do Ensino Médio: na forma de introdução conceitual para o segundo ano e como ferramenta para resolução de problemas no terceiro ano. Podemos dizer, como Malta (2008, p.11), que "a Teoria dos Grafos apresenta aspectos pertinentes que merecem espaço no currículo da Escola Básica". Contudo, para que essa abordagem possa ser efetivada de forma adequada, é importante que os professores tenham conhecimento específico e pedagógico acerca do tema em questão.

Alguns aspectos normativos reforçam as potencialidades de se abordar a Matemática Discreta e a Teoria dos Grafos no Ensino Médio em sala de aula. As Orientações Educacionais Complementares aos Parâmetros Curriculares Nacionais defendem que "estar formado para a vida significa mais do que reproduzir dados, denominar classificações ou identificar símbolos. Significa [...] enfrentar problemas de diferentes naturezas" (BRASIL, 2002, p. 9). Nesse sentido, estudar elementos da Matemática Discreta pode significar a diversificação da Matemática para além dos elementos algébricos, tão marcantes nos estudantes da Escola Básica. É importante destacar que as Orientações Curriculares para o Ensino Médio sugerem, explicitamente, a discussão da Teoria dos Grafos no Ensino Médio: 
No Ensino Médio, o termo "combinatória" está usualmente restrito ao estudo dos problemas de contagem, mas esse é apenas um de seus aspectos. Outros tipos de problemas poderiam ser trabalhados na escola - são aqueles relativos a conjuntos finitos e com enunciados de simples entendimento relativo, mas não necessariamente fáceis de resolver. Um exemplo clássico é o problema das pontes de Königsberg, tratado por Euler. (BRASIL, 2006, p. 94).

Em oficina realizada durante o Encontro de Interação do Multicurso Matemática³, em agosto de 2012, aplicou-se um questionário exploratório com 94 professores de ensino médio da rede estadual do Espírito Santo. Constatou-se, por meio deste questionário, que 77\% dos professores presentes não estudaram Grafos durante sua formação inicial e 87\% nunca abordaram este conteúdo durante suas aulas de matemática. É importante destacar que alguns dos professores que responderam positivamente às perguntas do questionário estavam confusos entre o termo "grafos" e "gráficos". Dessa forma, a quantidade de professores que não estudaram Teoria dos Grafos em sua formação inicial pode ultrapassar três quartos da quantidade de entrevistados para a pesquisa. Nessa oportunidade, verificouse também que o desconhecimento da teoria e de atividades que contemplem esse conteúdo são os principais argumentos utilizados pelos professores que não abordam Grafos em suas aulas.

Este artigo é resultado de pesquisa de iniciação científica que está inserida no Projeto "Investigações sobre atividades didáticas desenvolvidas para o Pibid no Laboratório de Matemática do Ifes - Campus Vitória”. Nosso principal objetivo é investigar atividades que podem abordar grafos em nível de Educação Básica, desenvolver materiais e sequências didáticas para serem apresentadas em oficinas e aplicadas em salas de aula.

O objetivo deste trabalho foi discutir o problema das sete pontes de Konisberg juntamente com uma releitura do problema associado à cidade de Vitória do Estado do Espírito Santo Brasil, uma vez que a capital capixaba também tem sua parte insular. Dessa forma, pretendemos apresentar uma atividade que permite ao professor de matemática iniciar uma

\footnotetext{
30 Multicurso é um programa de formação continuada, desenvolvido pela Fundação Roberto Marinho, que mescla educação presencial, por meio de grupos de estudos e encontros de interação, com a aprendizagem em rede, em plataformas virtuais.
} 
discussão sobre conceitos básicos da Teoria dos Grafos, como vértice e aresta, além do Teorema da existência de caminhos eulerianos.

\section{PRESSUPOSTOS TEÓRICOS E METODOLÓGICOS}

Ao realizar estudo das principais obras da Teoria dos Grafos, procuramos levantar atividades que poderiam contemplar este tema em nível de educação básica, já que nosso objetivo baseia-se em fornecer ao professor do ensino médio atividades que permitam uma abordagem inicial da Teoria dos Grafos. Com este direcionamento, localizamos a produção de Jurkiewicz (2007), Lopes (2010) e Fomin, Genkin e Itenberg (2010), que apresentam atividades direcionadas à educação básica. Contudo, estas atividades não contemplavam nosso objetivo inicial, tendo em vista a necessidade de mediação do professor durante a realização da proposta. Buscando atividades discutidas em pesquisas, encontramos trabalhos orientados pela metodologia de Resolução de Problemas (MALTA, 2008), de Atividades Investigativas (DEGGERONI, 2010; GUALANDI, 2012) e de Modelagem Matemática (MUNIZ JUNIOR, 2007). Inicialmente, realizamos estudo piloto a fim de refletir sobre o uso dessas metodologias (SÁ; PALMEIRA, 2012), mas, foi a partir da utilização de história da matemática (SÁ; SILVA, 2012a; SÁ; SILVA, 2012b) que passamos a vislumbrar, então, a oportunidade de se abordar a Teoria dos Grafos nessa perspectiva.

Acreditamos que a história da matemática é uma das metodologias que podem ser utilizadas na abordagem de Grafos no Ensino Médio, pois pode contribuir para que o aluno perceba a matemática como uma produção humana. Reafirmando esse pensamento, as Orientações Educacionais Complementares aos Parâmetros Curriculares Nacionais (PCN+) afirmam que

a importância da história das Ciências e da Matemática, contudo, tem uma relevância para o aprendizado que transcende a relação social, pois ilustra também o desenvolvimento e a evolução dos conceitos a serem aprendidos (BRASIL, 2002, p. 54). 
Em particular, Swetz (1989, apud MIGUEL, 1997, p. 81) apresenta outros argumentos reforçadores das potencialidades pedagógicas da história da matemática e sugere o uso de problemas históricos, pois estes

1) possibilitam o esclarecimento e o reforço de muitos conceitos que estão sendo ensinados; 2) constituem-se em veículos de informação cultural e sociológica; refletem as preocupações práticas ou teóricas de diferentes culturas em diferentes momentos históricos;

3) constituem-se em meio de aferimento da habilidade matemática de nossos antepassados;

4) permitem mostrar a existência de uma analogia ou continuidade entre os conceitos e processos matemáticos do passado e do presente.

Embora haja na história da Teoria dos Grafos importantes problemas, escolhemos o Problema das Sete Pontes de Konisberg por este ser o problema disparador da teoria e também pelo fato de que Vitória, assim como a cidade russa, tem sua parte insular com pontes de acesso ao continente. Essa estratégia de aproximar o problema histórico a um problema com dados reais e próximos ao cotidiano dos alunos. Também sobre o uso adaptado de problemas históricos, Brito e Carvalho (2009, p. 17) enfatizam que

Tais problemas não são obrigatoriamente os mesmos que os encontrados na História da Matemática, mas recriações destes. Outra finalidade do uso da história foi discutir procedimentos diferentes que possuem certe hegemonia no ensino de Matemática.

A atividade apresentada neste artigo foi realizada em uma turma de segundo ano do Ensino Médio, composta por vinte e seis alunos de uma escola pública situada em Vitória-ES. A dinâmica, na qual esta atividade está inserida, foi realizada em três aulas do turno matutino, entre os dias 04 e 05 de junho de 2012, e contou com o auxílio de três bolsistas do Pibid, além da professora regente da classe. A dinâmica adotada foi a de exibição de um vídeo informativo ${ }^{4}$, resolução de problemas e apresentação de soluções dos problemas sugeridos. Destacaremos o Problema das seis pontes de Vitoria e as estratégias dos alunos durante a resolução.

\footnotetext{
4 Vídeo informativo sobre resolução de problemas. Disponível em: <http://m3.ime.unicamp.br/recursos/1060>. Acesso em: 02/06/2012.
} 


\section{BREVE HISTÓRICO DA TEORIA DOS GRAFOS}

No início do século XVIII, especula-se que os cidadãos da cidade russa de Königsberg costumavam passar suas tardes de domingo caminhar em torno de sua cidade. A própria cidade consistiu em quatro áreas de terra separadas pelo Pregel sobre a qual há sete pontes, tal como ilustrado na Figura 1. O problema que os cidadãos fixaram era caminhar ao redor da cidade, cruzando cada uma das sete pontes apenas uma vez e, se possível, retornar ao seu ponto de partida.

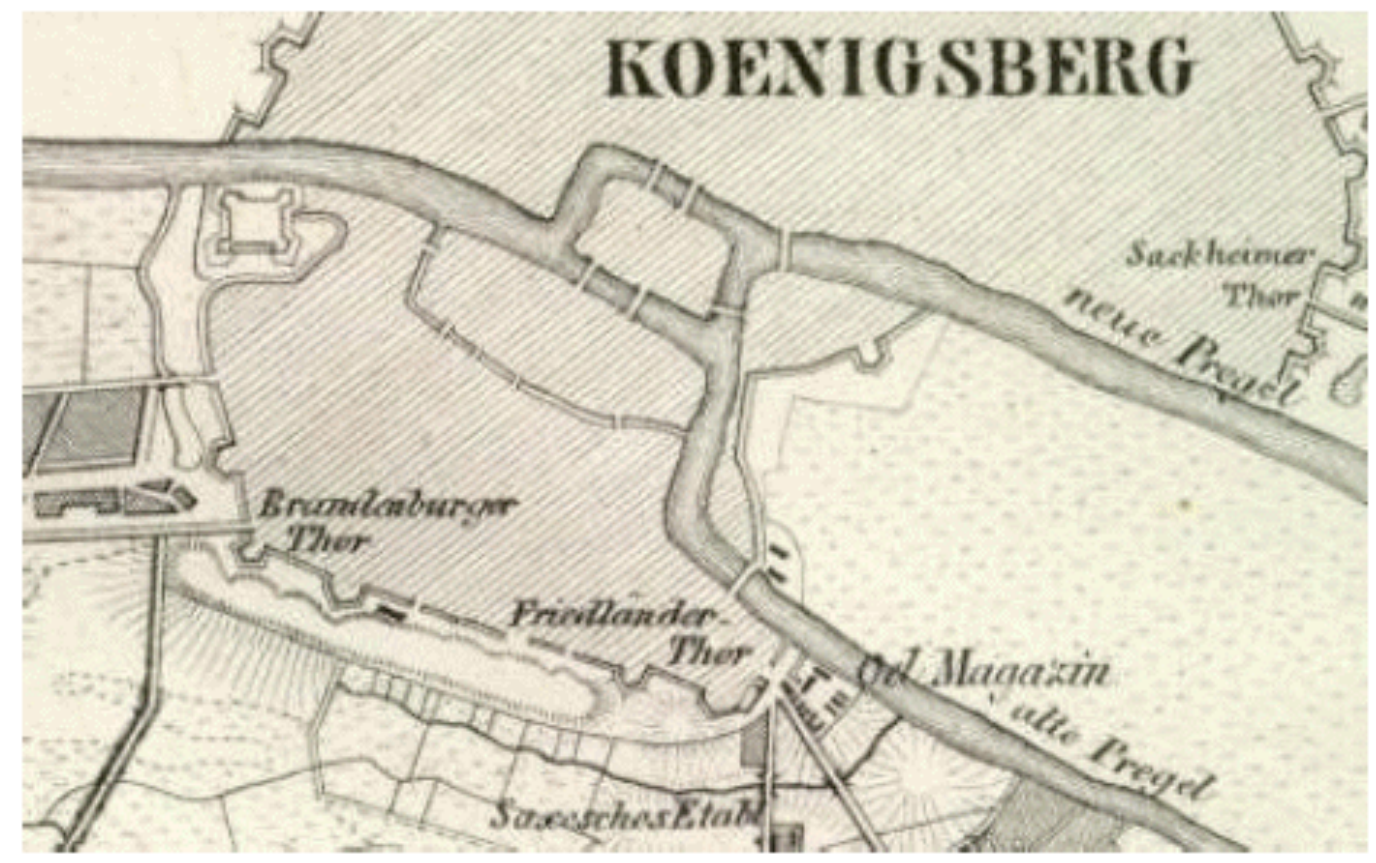

Figura 1: Mapa com as Pontes de Konisberg.

Em 1730, Leonhard Euler chega à Rússia para ocupar a cadeira de Filosofia Natural na Academia de Ciências de São Petersburgo. Três anos mais tarde, com a saída Daniel Bernoulli, ele tornou-se o principal matemático da Academia, que, nessa época, tinha lançado uma revista de matemática, chamada de Commentarii Academia e Scientiarum Imperialis Petropolitanae. Esta revista foi, durante muito tempo, abastecida com contribuições de Euler. Segundo Boyer (1974, p. 324), “os editores não tinham que se preocupar com a falta de material enquanto a pena de Euler trabalhasse". 
Após tomar conhecimento da notoriedade de Euler, em 1736, o prefeito da cidade de uma cidade próxima a Könisberg enviou uma carta a Eulerem nome de Heinrich Kiihn, um professor de matemática local. As mensagens trocadas inicialmente não foram recuperadas, mas uma carta datada de 09 de março indica que eles haviam discutido o problema. Um trecho dessa carta enviada a está apresentada da seguinte forma:

[...] você prestaria a mim e a nosso amigo Kiihn o mais valioso serviço, colocando-nos muito em dívida com você, culto Senhor, se você nos enviasse a solução, que você conhece bem, para o problema das sete pontes Könisberg, juntamente com uma prova. [...] eu adicionei um esboço das referidas pontes [...] (SACHS; STIEBITZ; WILSON, 1988, p. 134) ${ }^{5}$.

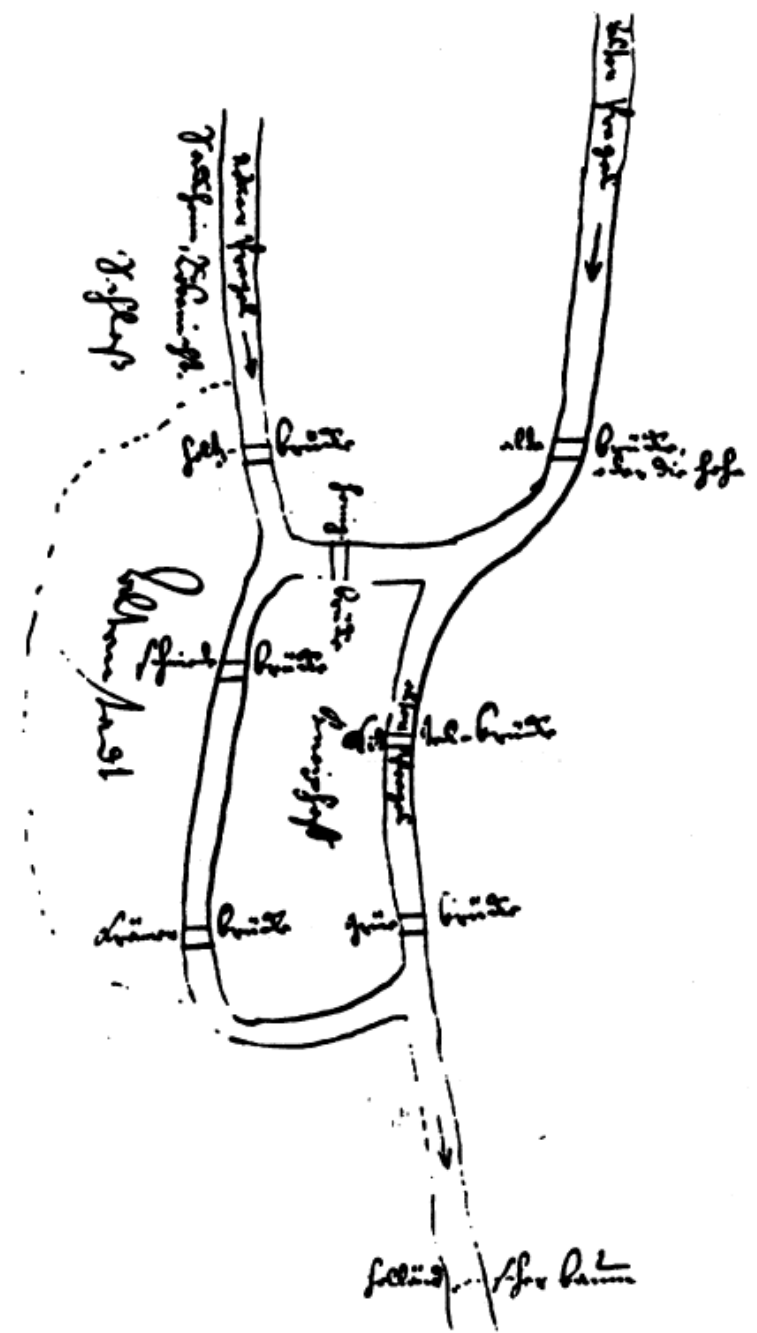

Figura 2: Esboço do mapa da cidade enviado a Euler

Fonte: Sachs, Stiebitz e Wilson (1988, p. 135).

\footnotetext{
5 Tradução livre de "You would render to me and our friend Kiihn a most valuable service, putting us greatly in your debt, most learned Sir, if you would send us the solution, which you know well, to the problem of the seven Kinigsberg bridges, together with a proof. [...] I have added a sketch of the said bridges...".
} 


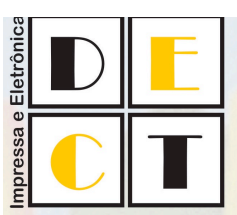

Quatro dias após receber essa última mensagem do prefeito, Euler escreveu a Giovanni Marinoni (Figura 3), um matemático e engenheiro italiano que morava em Viena:

Um problema me foi apresentado sobre uma ilha na cidade de Konigsberg, cercada por um rio, atravessado por sete pontes, e foi me perguntado se alguém poderia atravessar as pontes separadas em uma caminhada contínua de tal forma que cada ponte fosse atravessada apenas uma vez. Fui informado que até então ninguém havia demonstrado a possibilidade de fazer isso, ou mostrado que é impossível. Esta questão é tão banal, mas pareceu-me digno de atenção em que nem a geometria, álgebra, ou mesmo a arte de contar foram suficientes para resolvê-lo (HOPKINS; WILSON, 2004, p. 202) ${ }^{6}$.

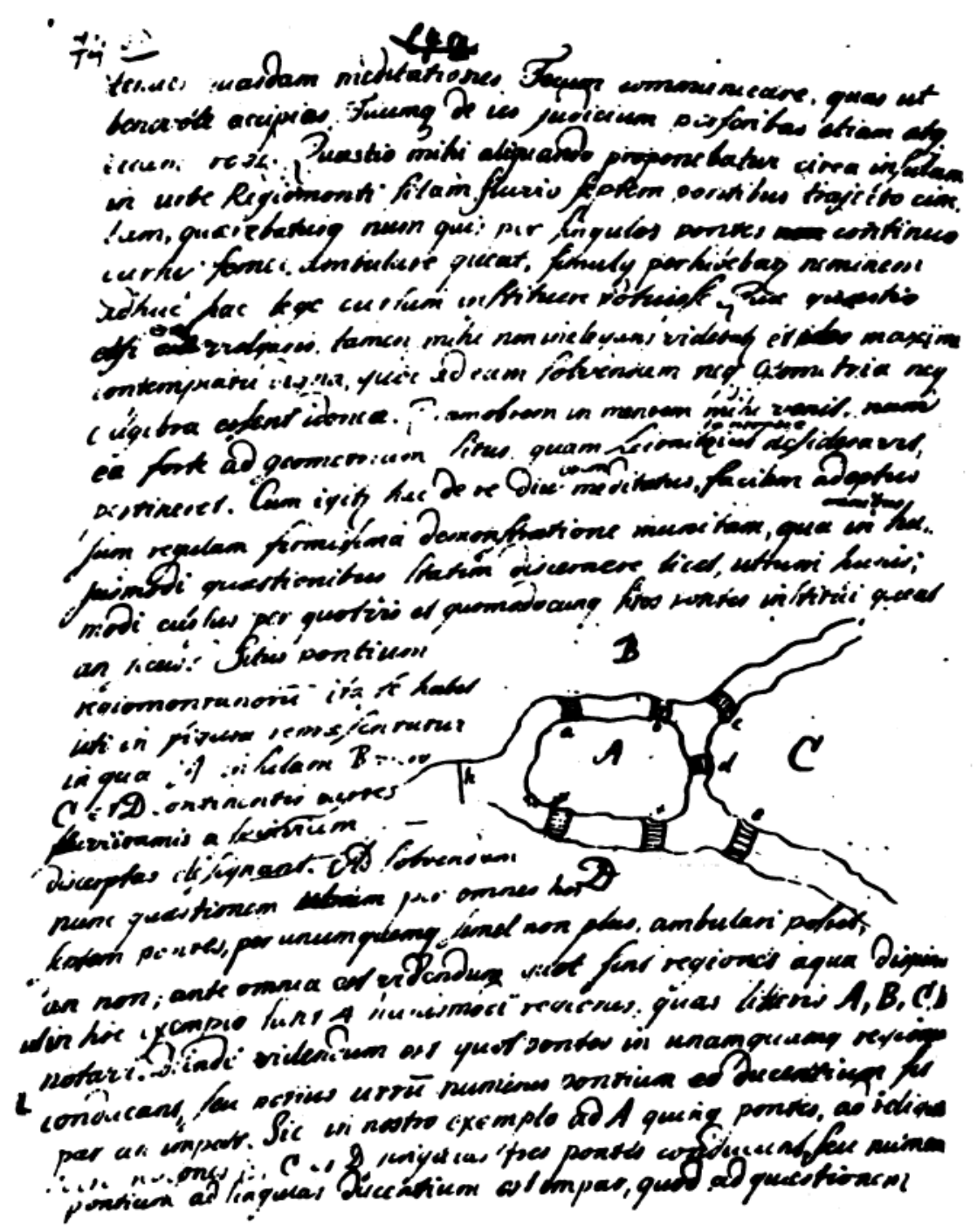

Figura 3: Carta de Euler para Marinoni

Fonte: Hopkins e Wilson (2004, p. 203).

6Tradução livre de "A problem was posed to me about an island in the city of Konigsberg, surrounded by a river spanned by seven bridges, and I was asked whether someone could traverse the separate bridges in a connected walk in such a way that each bridge is crossed only once. I was informed that hitherto no-one had demonstrated the possibility of doing this, or shown that it is impossible. This question is so banal, but seemed to me worthy of attention in that geometry, nor algebra, nor even the art of counting was sufficient to solve it". 


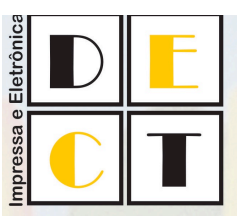

Verificamos, então, que a partir do Problema das Pontes de Konisberg, Euler percebeu a necessidade de sistematizar um novo campo da Matemática - era o surgimento da Teoria dos Grafos. Euler não precisou de mais de uma quinzena para resolver o enigma. No dia 03 de abril, o matemático enviou a resposta ao prefeito da cidade próxima a Konisberg, apresentando sua solução para o problema:

[...] Assim você vê, mais nobre senhor, como este tipo de solução tem pouca relação com a matemática, e eu não entendo por que você espera que um matemático para produzi-lo, ao invés de qualquer outra pessoa, já que a solução baseia-se na razão e sua descoberta não depende de qualquer princípio matemático. Devido a isso, eu não sei por que questões comuns que têm tão pouca relação com a matemática são resolvidos mais rapidamente pelos matemáticos do que por outros (HOPKINS; WILSON, 2004, p. 201)7.

Para solucionar o problema, Euler criou um desenho que esboçasse a cidade. Para tanto, ele representou as pontes por arestas e as porções de terra (ilhas e margens) por vértices, conforme ilustrados nas Figuras 5 e 6 . Assim, foi construído o primeiro grafo ${ }^{8}$ da história.

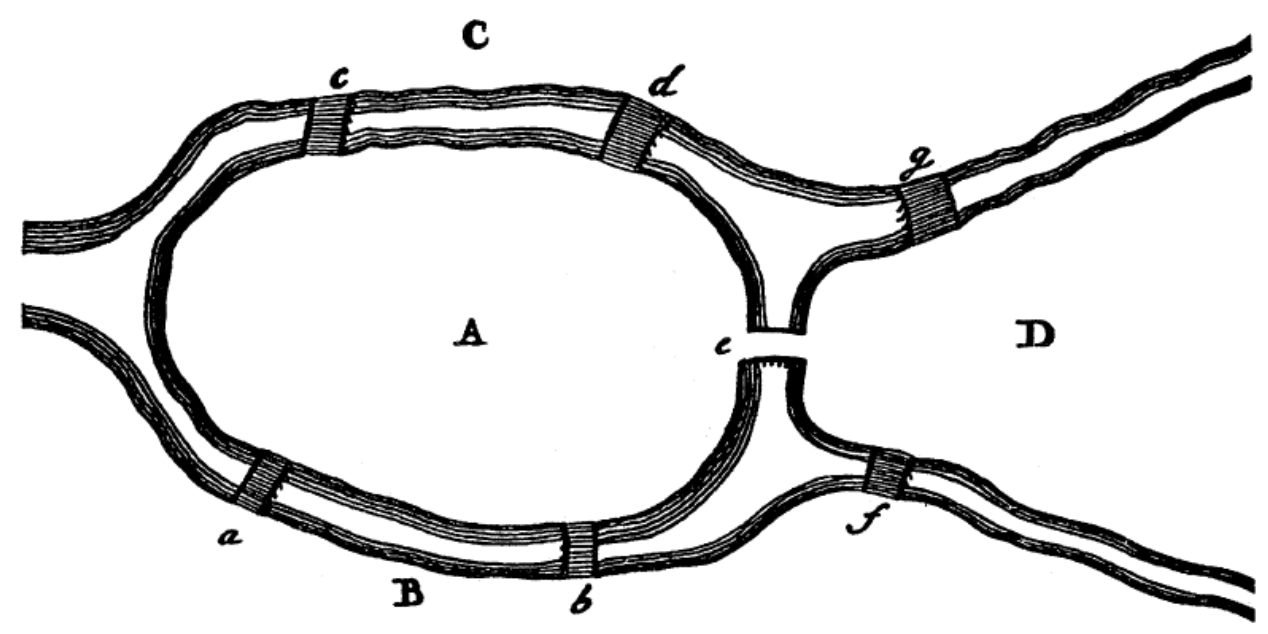

Figura 4: Diagrama utilizado por Euler em seu artigo (1736).

\footnotetext{
7Tradução livre de "Thus you see, most noble Sir, how this type of solution bears little relationship to mathematics, and I do not understand why you expect a mathematician to produce it, rather than anyone else, for the solution is based on reason alone, and its discovery does not depend on any mathematical principle. Because of this, I do not know why even questions which bear so little relationship to mathematics are solved more quickly by mathematicians than by others".

${ }^{8}$ É importante ressaltar também que "o termo grafo só foi utilizado pela primeira vez por Sylvester em 1878" (BOAVENTURA NETTO, 2006, p. 2).
} 


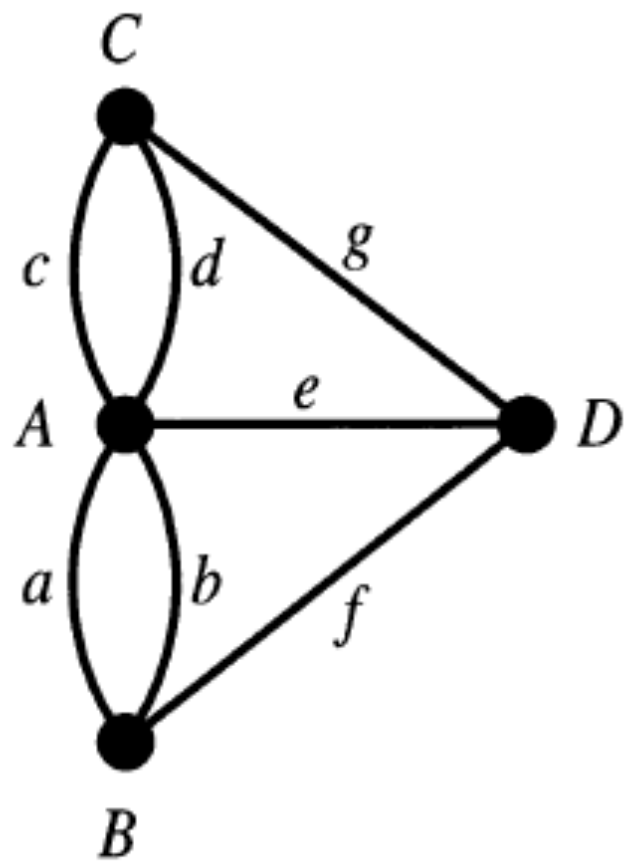

Figura 5: Grafo que representa o Problema das Sete Pontes de Konisberg.

Após chamar cada linha de aresta e cada ponto de vértice, Euler passou a analisar o grau de cada vértice. 0 que se procurava, então, era o que hoje chamamos de caminho euleriano fechado (no qual os pontos inicial e final coincidem). Determinamos um caminho desse tipo por meio do Teorema enunciado a seguir.

Teorema: Um grafo conexo admite caminho euleriano se, e somente se, todos os vértices têm grau par'

Segundo Lóvasz, Pelikán e Vesztergombi (2005), em 1736, Euler argumentou que se um nó $v$ tem grau ímpar, então todo passeio euleriano tem que começar ou terminar em $v$. Igualmente, se um nó $v$ tem grau par, então todo passeio euleriano começa e termina em $v$, ou começa e termina em outro lugar. Como esta justificativa pode não se fazer entender pelo leitor, apresentamos uma prova mais simplificada, baseada na demonstração de Deggeroni (2010).

\footnotetext{
${ }^{9}$ É importante destacar que o referencial adotado nesta pesquisa também considera a existência de caminhos semi-eulerianos que se caracterizam pela existência de exatamente dois vértices de grau ímpar.
} 


\section{Prova:}

$\Leftrightarrow$ ) Seja G um grafo que contém um caminho euleriano. Por cada ocorrência de vértice desse passeio, existe uma aresta que chega nesse vértice e outra que sai desse vértice. Como toda aresta faz parte do passeio e não há repetição de arestas no passeio, necessariamente, o número de arestas incidentes em cada vértice $v$ é igual a $2 \mathrm{k}$, onde $\mathrm{k}$ representa a quantidade de vezes que o passeio passa por $v$.

$(\Leftarrow)$ Como todo vértice possui grau par, então na construção de caminhos é sempre possível chegar e sair de um vértice e retornar a ele por arestas diferentes ainda não utilizadas. Assim, é possível sair de um vértice $v$ e retornar a ele sem repetição de arestas, conforme enunciado.

Retomando problema das sete pontes de Konisberg, verificamos no grafo da Figura 1 que $g(A)=5, g(B)=3, g(C)=3$ e $g(D)=3$. Assim, concluímos que há mais de dois vértices com grau ímpar e, por isso, não é possível estabelecer nenhum tipo de caminho por todas as pontes de Konisberg.

Para divulgar sua solução a comunidade científica da época, Euler escreveu a sua solução em seu artigo célebre no Commentarii Academiae Scientiarum Imperialis Petropolitanae sob o título "Solutio problematis ad geometriam situs pertinentis". 0 artigo (EULER, 1851) é dividido em vinte e um parágrafos numerados, dos quais o primeiro atribui o problema à geometria da posição ${ }^{10}$, os próximos oito são dedicados à solução do Problema das Sete Pontes de Könisberge os demais generalizam o problema. Embora datado de 1736, o jornal de Euler só foi publicado em 1741, com reimpressão em 1752.

Após a resolução do Problema das Sete Pontes de Könisberg, começaram a surgir novos problemas, como o do Caixeiro Viajante, o das Quatro Cores e o do Carteiro Chinês, que permitiram o desenvolvimento da Teoria dos Grafos. Ainda assim, o problema de Euler e o de Hamilton, que foi discutido anos depois, formam os principais problemas históricos com Grafos.

10 Até o Século XIX, este problema estava associado à Geometria da Posição. Contudo, esta geometria, segundo Debuiche (2012), não possui grandezas, números ou quantidades, o que desvincula os Grafos dessa Teoria. 


\section{O PROBLEMA DAS SEIS PONTES DE VITÓRIA}

No problema que foi proposto aos estudantes do ensino médio, os alunos do curso de Arquitetura e Urbanismo da Universidade Federal do Espírito Santo (Ufes) estão realizando um trabalho sobre as pontes de Vitória. Para isso, elas precisariam atravessar as seis pontes que dão acesso à capital capixaba. Dessa forma, os estudantes do ensino médio deveriam observar um mapa (Figura 7), como o que é apresentado abaixo, e verificar se era possível que o grupo de universitários realize algum trajeto que contemple todas as pontes que serão estudadas de forma que não repita a travessia de nenhuma ponte. Os alunos também deveriam considerar que os alunos da Ufes partiriam da Universidade.

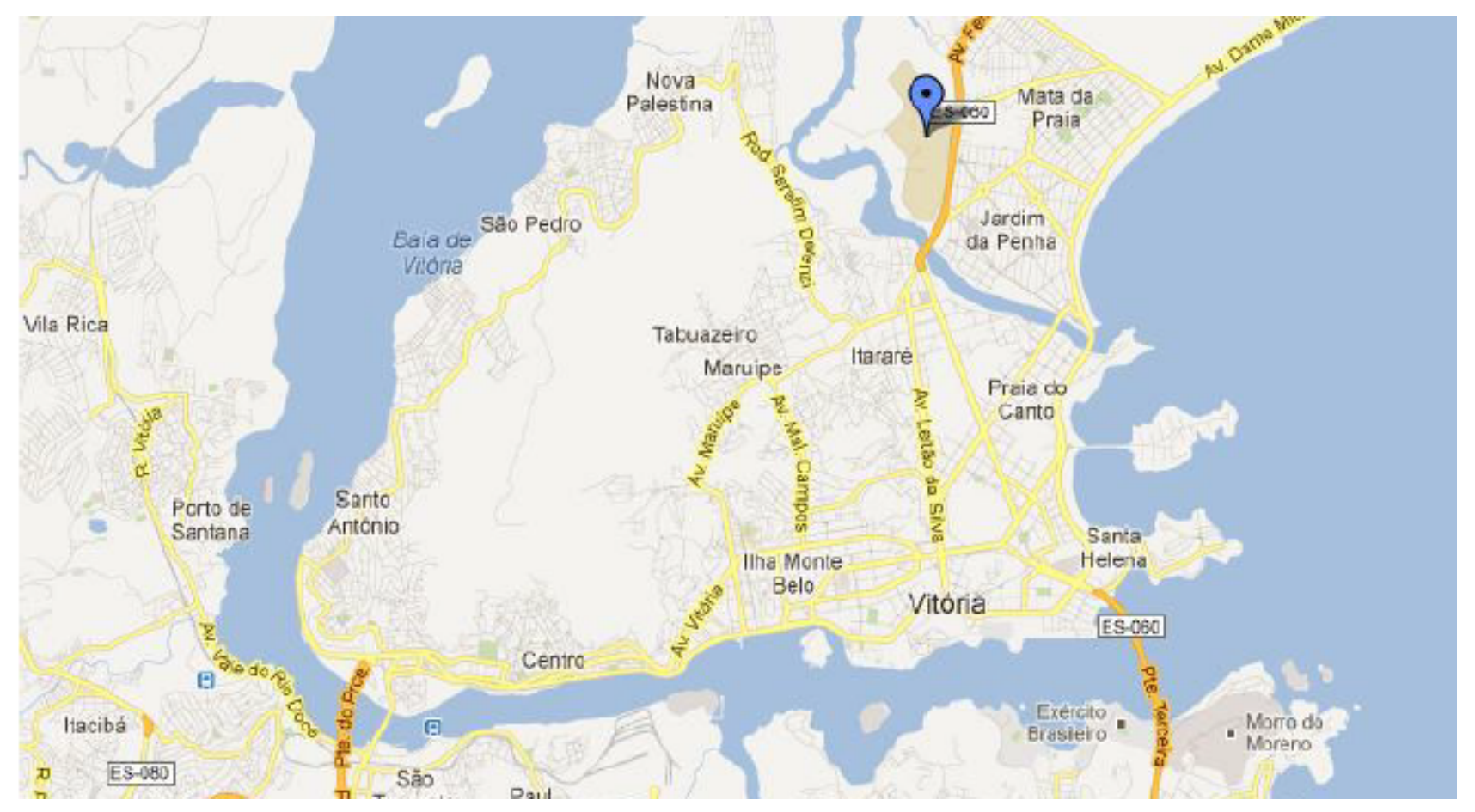

Figura 6. Ilha de Vitória com marcador onde está localizada a Ufes

Fonte: GoogleMaps.

Assim como Euler fez no problema das pontes de Konisberg, podemos esboçar um grafo que represente a região da Grande Vitória para que em seguida possamos verificar se esse grafo possui um caminho euleriano que inicie da porção de terra mais ao norte, onde está localizada a Ufes. 
No esboço do grafo, representamos as margens e a ilha central por vértices e as pontes por arestas. Chamamos os vértices que representam as margens superior e inferior, respectivamente, de A e C e ficamos, então, com a ilha-capital B. Como podemos observar no mapa, a ilha B está ligada a cada uma das margens por meio de três pontes. Assim, obtemos a representação apresentada na Figura 8.

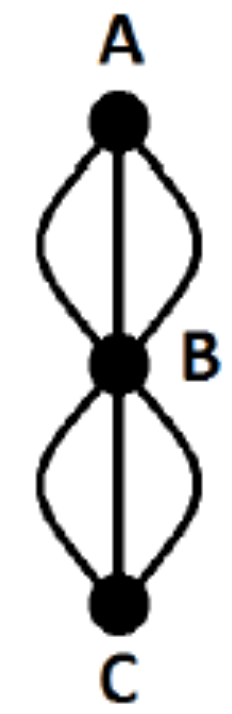

Figura 7: Grafo que representa a região da Grande Vitória.

Analisando o grafo acima, temos que $G(A)=3, G(B)=6$ e $G(C)=3$. Considerando o a discussão feita sobe Caminhos Euleriano e Semieulerianos, verificamos que essa situação leva a um caminho do segundo tipo. Contudo, o fato do caminho ser aberto não é problema, pois conforme restringido inicialmente, o caminho deverá iniciar-se no vértice A, que possui grau ímpar e que representa a margem onde se encontra a Ufes. Concluímos assim que há um trajeto que contemple todas as pontes que serão estudadas de forma que não repita a travessia de nenhuma ponte. No entanto, os universitários não terminarão o percurso na Ufes, pois o caminho euleriano apresentado não é fechado.

\section{CONSIDERAÇÕES FINAIS}

Acreditamos que o assunto Teoria dos Grafos pode ser ensinado no Ensino Médio bastando que os professores saibam abordar esse tema utilizando problemas próximos da realidade e que façam sentido para os alunos. Ressaltamos que a abordagem dessa Teoria pode ser 
realizada em diversos momentos, mas procuramos nesse primeiro contato verificar a aceitação de tal assunto pelos alunos do Ensino Médio. Ao realizar essa pesquisa bibliográfica verificamos a importância de um resgate histórico para compreendermos o desenvolvimento dessa Teoria dos Grafos. Ressaltamos que o acesso a algumas cartas originais proporcionou esclarecimentos sobre o contexto de formulação dessa teoria. 0 conhecimento dessa história contribuiu para a organização e produção da atividade proposta sobre a releitura para a cidade de Vitória.

O problema das pontes de Vitória foi o que mais provocou os alunos durante a realização da dinâmica. Acreditamos que a forma com que foi proposto o problema e a proximidade com a realidade contribuiu para a grande aceitação e engajamento dos alunos frente à proposta apresentada. Durante a correção das atividades, verificamos que o grupo responsável pela resolução desse problema havia interpretado as pontes como vértices. Assim, o grafo esboçado era diferente do correto. Como o esboço de grafo apresentado não permitia uma análise que respondesse à pergunta, convidamos a classe para solucionar o problema proposto. A turma conseguiu identificar quais elementos seriam representados por vértices e quais seriam pelas arestas. Em discussão, concluímos que o problema proposto inicialmente não havia solução, pois o ideal é que percursos como o proposto no problema devem terminar no ponto de partida.

Foi possível estabelecer relações entre estratégias de resolução de alunos e do matemático Euler. Verificamos que alguns alunos apresentaram rudimentos do Teorema da Existência do Caminho Euleriano e, além disso, um aluno enunciou o teorema durante a resolução do Problema das Pontes de Vitória. Com efeito, essas cartas foram utilizadas como disparador da discussão da solução de Euler. Nesse sentido, acreditamos ter evidenciado que a resolução de um aluno de Ensino Médio não possui menor valor que a de um matemático.

A experiência vivenciada neste trabalho nos leva a refletir que a proposta de se trabalhar a Teoria dos Grafos no Ensino Médio é válida. Verificamos que a História da Matemática pode ser uma metodologia utilizada para abordagem desse tema, pois foi a partir da apresentação do problema histórico que conseguimos inserir a abordagem realizada. 


\section{AGRADECIMENTOS}

Os autores agradecem ao Instituto Federal do Espírito Santo (Ifes) e à Fundo de Apoio à Ciência e Tecnologia do Município de Vitória (Facitec) pela oportunidade de realizar a Pesquisa de Iniciação Científica. Agradecemos também aos colegas do Grupo de Pesquisa em Práticas Pedagógicas de Matemática e do Grupo de Estudos em Educação Matemática do Espírito Santo pelas discussões e contribuições no decorrer da pesquisa. Gostaríamos de registrar nossos agradecimentos a Lee Stemkoski (Mathematical Association of America) pelo compartilhamento das cartas de Euler.

\section{REFERÊNCIAS}

BOAVENTURA NETTO, P. O.. Grafos: Teoria, Modelos e Algoritmos. 4.ed. São Paulo: E. Blücher, 2006.

BRASIL, PCN + Ensino Médio: Orientações Educacionais Complementares aos Parâmetros Curriculares Nacionais: Ciências da Natureza, Matemática e suas Tecnologias. MEC-SEMTEC: Brasília, 2002.

BRASIL, Orientações curriculares para o Ensino Médio: Ciências da natureza, matemática e suas tecnologias. Brasília: MEC/SEF, 2006.

BRITO, A. J.; CARVALHO, D. L.. Utilizando a história da no ensino de Geometria. In: MIGUEL, A. et al. História da Matemática em atividades didáticas. São Paulo: Editora da Física, 2009.

CHARTAND, G. Introductory Graph Theory. Boston: DoverPublications, 1977.

DEBUICHE, V. Leibniz's Manuscripts on Perspective. In: 5th International Conference European Society of the History of Science. Athenas, 2012.

DEGGERONI, R.Uma introdução à teoria dos Grafos no Ensino Médio. Trabalho de Conclusão de Curso (Licenciatura em Matemática) - Universidade Federal do Rio Grande do Sul, Porto Alegre, 2010.

ESPÍRITO SANTO (ESTADO). Secretaria da Educação. Currículo Básico Escola Estadual Ensino médio: área de Ciências da Natureza. Vitória: SEDU, 2009. 
EULER, L. Solution d'unproblèmeappartenant à lagéométrie de situation. NouvellesAnnalesdeMathématiques,1 série, tome 10 (1851), p. 106-119. Traduzido do Latim por E. Coupy.

FOMIN, D.; GENKIN, S.; ITENBERG, I..Círculos matemáticos: a experiência russa. Tradução de Valéria Magalhães Iório. Rio de Janeiro: IMPA, 2010.

GUALANDI, J; H. Investigações matemáticas com grafos para o ensino. Dissertação (Mestrado) - Pontifícia Universidade Católica de Minas Gerais. Programa de Pós-Graduação em Ciências e Matemática. Belo Horizonte, 2012.

HOPKINS, B.; WILSON, R. J..The truth about Konisberg. The College Mathematics Journal, vol. 35, n. 03, p. 198-207, mai, 2004.

JURKIEWICZ, S. Grafos: Uma introdução. Programa de Iniciação Científica da OBMEP 2007. N. 5. Parte integrante da coleção de 2007. Também disponível em <http://www.obmep.org.br/export/sites/default/arquivos/apostilas_pic2010/Apostila5-

Grafos.pdf $>$. Acesso em: 26 de maio de 2012.

LÓVASZ, L.; PELIKÁN, J.; VESZTERGOMBI K. Matemática discreta: elementar e além. Tradução de Ruy de Queiroz. Rio de Janeiro: SBM, 2005.

LOPES, M. L. M. L. (coord.) Grafos: jogos e desafios. Rio de Janeiro: IM/UFRJ, 2010.

LÓSS, Z. E..o Desenvolvimento da Pesquisa Operacional no Brasil. Dissertação (Mestrado em Engenharia de Produção) - Universidade Federal do Rio de Janeiro, Programa de Engenharia de Produção, Rio de Janeiro, 1981.

MALTA, G. H. S. Grafos no ensino médio: uma inserção possível. Dissertação (Mestrado em Ensino de Matemática) - Universidade Federal do Rio Grande do Sul, Programa de PósGraduação em Ensino de Matemática, Porto Alegre, 2008.

MIGUEL, A.. As potencialidades pedagógicas da história da matemática em questão: argumentos reforçadores e questionadores. ZETETIKÉ, Campinas/SP, v. 5, n. 8, Jul./Dez. 1997. p.73-105.

MUNIZ JUNIOR, I. Encontrando, minimizando e planejando percursos: uma introdução à teoria dos grafos no ensino médio. 134f. Dissertação (Mestrado) - Centro Federal de Educação Tecnológica Celso Suckow, Programa de Pós-Graduação em Ensino de Ciências e Matemática, Rio de Janeiro, 2007.

ORE, O. Graphs e their uses. Washington: The Mathematical Association of America, 1990.

SÁ, L. C. ; PALMEIRA, C. A. . Primeiras atividades didáticas para o ensino de Grafos no Ensino Médio. In: Anais daIII Jornada de Iniciação à Docência. Vitória, 2012. 
SÁ, L. C.; SILVA, S. A .F. Uso de problema histórico para abordagem de Grafos no Ensino Médio. In: Anais da VII Jornada de Iniciação Científica, Desenvolvimento Tecnológico e Inovação do Ifes. Serra, 2012a.

De Konisberg a Vitória: o problema das pontes da capital capixaba em uma atividade didática sobre grafos. In: Anais da VI Bienal da Sociedade Brasileira de Matemática. Campinas, 2012b.

SACHS, H.; STIEBITZ, M.; WILSON, R. J.. An historical note: Euler's Konisberg Letters. Journal of Graph Theory , vol. 12, n. 01, p. 133-139, 1988. 